\title{
The Fourth Fundamental Form IV of Dini-Type Helicoidal Hypersurface in the Four Dimensional Euclidean Space
}

\author{
Erhan Güler (iD
}

check for updates

Citation: Güler, E. The Fourth Fundamental Form $I V$ of Dini-Type Helicoidal Hypersurface in the Four Dimensional Euclidean Space . Axioms 2021, 10, 186. https:// doi.org/10.3390/axioms10030186

Academic Editors: Anna Maria Fino and Hari Mohan Srivastava

Received: 8 July 2021

Accepted: 12 August 2021

Published: 16 August 2021

Publisher's Note: MDPI stays neutral with regard to jurisdictional claims in published maps and institutional affiliations.

Copyright: (C) 2021 by the author. Licensee MDPI, Basel, Switzerland. This article is an open access article distributed under the terms and conditions of the Creative Commons Attribution (CC BY) license (https:/ / creativecommons.org/licenses/by/ $4.0 /)$.
Department of Mathematics, Faculty of Science, Kutlubey Campus, Bartın University, 74100 Bartın, Turkey; eguler@bartin.edu.tr; Tel.: +90-378-5011000 (ext. 1791)

Abstract: We introduce the fourth fundamental form of a Dini-type helicoidal hypersurface in the four dimensional Euclidean space $\mathbb{E}^{4}$. We find the Gauss map of helicoidal hypersurface in $\mathbb{E}^{4}$. We obtain the characteristic polynomial of shape operator matrix. Then, we compute the fourth fundamental form matrix $I V$ of the Dini-type helicoidal hypersurface. Moreover, we obtain the Dini-type rotational hypersurface, and reveal its differential geometric objects.

Keywords: four dimension; Dini-type helicoidal hypersurface; Gauss map; shape operator; curvatures; fourth fundamental form

MSC: Primary: 53A07; Secondary: 53C42

\section{Introduction}

Rotational and helicoidal hyper-surfaces have attracted the attention of scientists such as architects, biologists, physicists, mathematicians, and especially geometers for almost 300 years.

Let us review some works about rotational and helicoidal characters in chronological order.

Catenoid is a minimal rotational surface described by Euler [1] in 1744. Helicoid is a ruled minimal surface, described by Euler in 1774 and by Meusnier [2] in 1776. Bour [3] gave isometric deformation formulas of catenoid-helicoid. Dini [4] obtained a helicoidal surface.

Moore [5] introduced rotational surfaces in a four dimensional space $\mathbb{E}^{4}$. Moore [6] considered rotational surfaces of constant curvature in $\mathbb{E}^{4}$.

Do Carmo and Dajczer [7] worked on helicoidal surfaces with constant mean curvature CMC. Chen [8] gave submanifolds of a finite type in his books. Hano and Nomizu [9] considered surfaces of revolution with CMC. Roussos [10] studied helicoidal surfaces as Bonnet surfaces. Ripoll [11] introduced helicoidal minimal surfaces in hyperbolic space. Dillen [12] worked on ruled submanifolds of finite type. Baikoussis and Verstraelen [13] focused on the Gauss map of helicoidal surfaces. Hoffman, Wei, and Karcher [14] considered adding handles to the helicoid. Gray [15] gave details of helicoidal-rotational surfaces in his book. Baikoussis and Koufogiorgos [16] obtained helicoidal surfaces with prescribed mean or Gaussian curvature. Dillen and Kühnel [17] examined ruled Weingarten surfaces in Minkowski 3-space.

Ikawa [18] introduced Bour's theorem and Gauss map. Sasahara [19] worked on spacelike helicoidal surfaces with CMC in a Minkowski 3-space. Ikawa [20] focused on Bour's theorem in Minkowski geometry. Choi and Kim [21] characterized the helicoid as a ruled surface with pointwise 1-type Gauss map. Yoon [22] studied rotational surfaces with finite type Gauss map in $\mathbb{E}^{4}$. Beneki, Kaimakamis, and Papantoniou [23] indicated the Minkowski 3-space of helicoidal surfaces. Güler and Vanl1 [24] considered Bour's theorem in Minkowski 3-space. Güler and Vanlı [25] classified the mean, Gaussian, 2nd Gaussian and the 2nd mean curvatures of the helicoidal surfaces with light-like axis in Minkowski 3-space. 
Stamatakis and Al-Zoubi [26] considered surfaces of a revolution satisfying $\Delta^{I I I} x=A x$. Ji and Kim [27] worked on helicoidal CDPC-surfaces in Minkowski 3-space. Ji and Kim [28] introduced mean curvatures and Gauss maps of a pair of isometric helicoidal and rotation surfaces in Minkowski 3-space. Güler, Yayl1, and Hacısalihoğlu [29] used Bour's theorem on the Gauss map in 3-Euclidean space. Arslan et al. [30] studied rotational embeddings with pointwise 1-type Gauss map in $\mathbb{E}^{4}$. Dursun and Turgay [31] worked on general rotational surfaces with pointwise 1-type Gauss map in $\mathbb{E}^{4}$. Arslan et al. [32] focused on generalized rotation surfaces in $\mathbb{E}^{4}$.

Dursun and Turgay [33] studied minimal and pseudo-umbilical rotational surfaces in Euclidean space $\mathbb{E}^{4}$. Perdomo [34] provided helicoidal minimal surfaces in $\mathbb{R}^{3}$. Ji and Kim [35] considered isometries between minimal helicoidal surfaces and rotation surfaces in Minkowski space. Kim and Turgay [36] introduced surfaces with $L_{1}$-pointwise 1-type Gauss map in $\mathbb{E}^{4}$. Kim and Turgay [37] classified helicoidal surfaces with $L_{1}$-pointwise 1-type Gauss map. Güler [38] introduced a new type of helicoidal surface of value $m$. López and Demir [39] worked on helicoidal surfaces in Minkowski space with CMC and CGC. Arslan, Bulca, and Milousheva [40] studied meridian surfaces with pointwise 1-type Gauss map in $\mathbb{E}^{4}$. Ganchev and Milousheva [41] considered general rotational surfaces in 4-dimensional Minkowski space. Babaarslan and Yaylı [42] gave space-like loxodromes on rotational surfaces in Minkowski 3-space. Güler and Yaylı [43] introduced generalized Bour's theorem. Senoussi and Bekkar [44] considered helicoidal surfaces with $\Delta^{J} r=A r$ in $\mathbb{E}^{3}$.

Hoffman, Traizet, and White [45] gave helicoidal minimal surfaces of a prescribed genus. Kim, Kim, and Kim [46] focused on the Cheng-Yau operator and Gauss map of surfaces of revolution. Güler, Magid, and Yaylı [47] focused on the Laplace Beltrami operator of a helicoidal hypersurface in four space. Hieu and Thang [48] considered Bour's theorem in 4-dimensional Euclidean space. Arslan, Bulca, and Kosova [49] studied generalized rotational surfaces in Euclidean spaces. Babaarslan and Kayacık [50] considered time-like loxodromes on helicoidal surfaces in Minkowski 3-space. Güler, Hacısalihoğlu, and Kim [51] gave the Gauss map and the third Laplace-Beltrami operator of the rotational hypersurface in 4-space. Aleksieva, Milousheva, and Turgay worked on [52] general rotational surfaces in pseudo-Euclidean 4-space with neutral metrics. Goemans [53] introduced flat double rotational surfaces in Euclidean and Lorentz-Minkowski 4-space. Güler and Turgay [54] obtained a Cheng-Yau operator and Gauss map of rotational hypersurfaces in 4-space. Güler and Kişi [55] indicated Dini-type helicoidal hypersurfaces with timelike axis in $\mathbb{E}_{1}^{4}$. Yoon, Lee, and Lee [56] constructed helicoidal surfaces by using curvature functions in isotropic space. Güler [57] worked helical hypersurfaces in $\mathbb{E}_{1}^{4}$.

Dursun [58] introduced rotational Weingarten surfaces in hyperbolic 3-space. Güler [59] focused on the fundamental form $I V$ and curvature formulas of the hypersphere. López and Pámpano [60] classified rotational surfaces with constant skew curvature in 3-space forms.

In this paper, we study the fourth fundamental form of the Dini-type helicoidal hypersurface in Euclidean 4-space $\mathbb{E}^{4}$. In Section 2, we offer some basic notions of fourdimensional Euclidean geometry. In Section 3, we define helicoidal hypersurface. In Section 4, we give Dini-type helicoidal hypersurface and calculate the fourth fundamental form. In addition, we provide a conclusion in the last section.

\section{Preliminaries}

In the rest of this paper, we identify a vector $(\mathrm{a}, \mathrm{b}, \mathrm{c}, \mathrm{d})$ with its transpose $(\mathrm{a}, \mathrm{b}, \mathrm{c}, \mathrm{d})^{t}$.

In this section, we will introduce the first, second, third, and fourth fundamental form matrices, matrix of the shape operator $S$ of hypersurface $\mathbf{x}=\mathbf{x}(u, v, w)$ in the fourdimensional Euclidean space $\mathbb{E}^{4}$.

Let $\mathbf{x}=\mathbf{x}(u, v, w)$ be an isometric immersion of any hypersurface $M^{3}$ in $\mathbb{E}^{4}$. Let $\left\{e_{1}, e_{2}, e_{3}, e_{4}\right\}$ be the standart base vectors of $\mathbb{E}^{4}$. The inner product of $\vec{x}=\left(x_{1}, x_{2}, x_{3}, x_{4}\right)$, $\vec{y}=\left(y_{1}, y_{2}, y_{3}, y_{4}\right)$, and the vector product of $\vec{x}, \vec{y}, \vec{z}=\left(z_{1}, z_{2}, z_{3}, z_{4}\right)$ on $\mathbb{E}^{4}$ are defined as follows 


$$
\begin{aligned}
\vec{x} \cdot \vec{y} & =x_{1} y_{1}+x_{2} y_{2}+x_{3} y_{3}+x_{4} y_{4} \\
\vec{x} \times \vec{y} \times \vec{z} & =\operatorname{det}\left(\begin{array}{cccc}
e_{1} & e_{2} & e_{3} & e_{4} \\
x_{1} & x_{2} & x_{3} & x_{4} \\
y_{1} & y_{2} & y_{3} & y_{4} \\
z_{1} & z_{2} & z_{3} & z_{4}
\end{array}\right)
\end{aligned}
$$

respectively.

In 4-space, the first and the second fundamental form matrices of hypersurface $\mathbf{x}(u, v, w)$ are given as follows

$$
I=\left(\begin{array}{lll}
E & F & A \\
F & G & B \\
A & B & C
\end{array}\right), I I=\left(\begin{array}{ccc}
L & M & P \\
M & N & T \\
P & T & V
\end{array}\right)
$$

where

$$
\begin{array}{lll}
E=\mathbf{x}_{u} \cdot \mathbf{x}_{u}, & F=\mathbf{x}_{u} \cdot \mathbf{x}_{v}, & G=\mathbf{x}_{v} \cdot \mathbf{x}_{v}, \\
A=\mathbf{x}_{u} \cdot \mathbf{x}_{w}, & B=\mathbf{x}_{v} \cdot \mathbf{x}_{w}, & C=\mathbf{x}_{w} \cdot \mathbf{x}_{w}, \\
L=\mathbf{x}_{u u} \cdot \mathcal{G}, & M=\mathbf{x}_{u v} \cdot \mathcal{G}, & N=\mathbf{x}_{v v} \cdot \mathcal{G}, \\
P=\mathbf{x}_{u w} \cdot \mathcal{G}, & T=\mathbf{x}_{v w} \cdot \mathcal{G}, & V=\mathbf{x}_{w w} \cdot \mathcal{G},
\end{array}
$$

and the Gauss map $\mathcal{G}=\mathcal{G}(u, v, w)$ of $\mathbf{x}$ is defined as follows

$$
\mathcal{G}=\frac{\mathbf{x}_{u} \times \mathbf{x}_{v} \times \mathbf{x}_{w}}{\left\|\mathbf{x}_{u} \times \mathbf{x}_{v} \times \mathbf{x}_{w}\right\|}
$$

Theorem 1. The shape operator matrix $S$ of any hypersurface $\mathbf{x}(u, v, w)$ in 4 -space is given as follows

$$
S=\frac{1}{\operatorname{det} I}\left(\begin{array}{lll}
s_{1} & s_{2} & s_{3} \\
s_{4} & s_{5} & s_{6} \\
s_{7} & s_{8} & s_{9}
\end{array}\right)
$$

where

$$
\begin{aligned}
& \operatorname{det} I=\left(E G-F^{2}\right) C-A^{2} G+2 A B F-B^{2} E, \\
& s_{1}=A B M-C F M-A G P+B F P+C G L-B^{2} L, \\
& s_{2}=A B N-C F N-A G T+B F T+C G M-B^{2} M, \\
& s_{3}=A B T-C F T-A G V+B F V+C G P-B^{2} P, \\
& s_{4}=A B L-C F L+A F P-B P E+C M E-A^{2} M, \\
& s_{5}=A B M-C F M+A F T-B T E+C N E-A^{2} N, \\
& s_{6}=A B P-C F P+A F V-B V E+C T E-A^{2} T, \\
& s_{7}=-A G L+B F L+A F M-B M E+G P E-F^{2} P, \\
& s_{8}=-A G M+B F M+A F N-B N E+G T E-F^{2} T, \\
& s_{9}=-A G P+B F P+A F T-B T E+G V E-F^{2} V .
\end{aligned}
$$

Proof. We compute $I^{-1}$.II, and it gives the shape operator matrix $S$.

Theorem 2. The third fundamental form matrix III of any hypersurface $\mathbf{x}(u, v, w)$ in 4-space is given as follows

$$
I I I=\frac{1}{\operatorname{det} I}\left(\begin{array}{ccc}
\Gamma & \Phi & \Omega \\
\Phi & \Psi & \Theta \\
\Omega & \Theta & \Delta
\end{array}\right),
$$

where $\Gamma=\left(\mathcal{G}_{u} \cdot \mathcal{G}_{u}\right) \operatorname{det} I, \Phi=\left(\mathcal{G}_{u} \cdot \mathcal{G}_{v}\right) \operatorname{det} I, \Omega=\left(\mathcal{G}_{u} \cdot \mathcal{G}_{w}\right) \operatorname{det} I, \Psi=\left(\mathcal{G}_{v} \cdot \mathcal{G}_{v}\right) \operatorname{det} I$, $\Theta=\left(\mathcal{G}_{v} \cdot \mathcal{G}_{w}\right) \operatorname{det} I, \Delta=\left(\mathcal{G}_{w} \cdot \mathcal{G}_{w}\right) \operatorname{det} I$, and 


$$
\begin{aligned}
\Gamma= & -A^{2} M^{2}-B^{2} L^{2}-F^{2} P^{2}+C G L^{2}+C E M^{2}+G E P^{2} \\
& +2(A B L M-E B M P-C F L M+A F M P-G A L P+B F L P), \\
\Phi= & A B M^{2}-C F M^{2}-B^{2} L M-A^{2} M N-F^{2} P T+C M N E \\
& -B N P E-B M T E+G P T E+A B L N-C F L N+C G L M \\
& +A F N P-A G M P+B F M P+A F M T-A G L T+B F L T, \\
\Omega= & -A G P^{2}-B^{2} L P+B F P^{2}-A^{2} M T-F^{2} P V+C M T E \\
& -B M V E-B P T E+G P V E+A B M P+A B L T-C F M P \\
& +C G L P-C F L T+A F M V-A G L V+B F L V+A F P T, \\
\Psi= & -A^{2} N^{2}-B^{2} M^{2}-F^{2} T^{2}+C G M{ }^{2}+C E N^{2}+G E T^{2} \\
& +2(A B M N+A F N T-G A M T+B F M T-E B N T-C F M N), \\
& A F T^{2}-B^{2} M P-A^{2} N T-F^{2} T V-B T^{2} E+C N T E \\
& -B N V E+G T V E+A B N P+A B M T-C F N P+C G M P \\
& -C F M T+A F N V-A G M V+B F M V-A G P T+B F P T, \\
& -A^{2} T^{2}-B^{2} P^{2}-F^{2} V^{2}+C G P^{2}+C E T^{2}+G E V^{2} \\
& +2(A B P T+A F T V-G A P V+B F P V-E B T V-C F P T) .
\end{aligned}
$$

Proof. We compute II.S, and this gives the matrix of the third fundamental form III.

Theorem 3. Matrix of the fourth fundamental form IV where the coefficients depends on I and II of a hypersurface $\mathbf{x}(u, v, w)$ in 4-space is given as follows

$$
I V=\frac{1}{(\operatorname{det} I)^{2}}\left(\begin{array}{ccc}
\alpha & \beta & \delta \\
\beta & \varepsilon & \xi \\
\delta & \xi & \eta
\end{array}\right),
$$

where

$$
\begin{aligned}
\alpha & =\Gamma s_{1}+\Phi s_{4}+\Omega s_{7}, \\
\beta & =\Gamma s_{2}+\Phi s_{5}+\Omega s_{8}=\Phi s_{1}+\Psi s_{4}+\Theta s_{7}, \\
\delta & =\Gamma s_{3}+\Phi s_{6}+\Omega s_{9}=\Omega s_{1}+\Theta s_{4}+\Delta s_{7}, \\
\varepsilon & =\Phi s_{2}+\Psi s_{5}+\Theta s_{8}, \\
\xi & =\Phi s_{3}+\Psi s_{6}+\Theta s_{9}=\Omega s_{2}+\Theta s_{5}+\Delta s_{8}, \\
\eta & =\Omega s_{3}+\Theta s_{6}+\Delta s_{9},
\end{aligned}
$$

Proof. We compute III.S, then it gives the fourth fundamental form matrix IV.

\section{Helicoidal Hypersurface}

Let $\gamma: I \longrightarrow \Pi$ be a curve in a plane $\Pi$ in $\mathbb{E}^{4}$, and let $\ell$ be a straight line in $\Pi$ for an open interval $I \subset \mathbb{R}$. A rotational hypersurface in $\mathbb{E}^{4}$ is defined as a hypersurface rotating a curve $\gamma$ (i.e., profile curve) around a line (i.e., axis) $\ell$. Suppose that when a profile curve $\gamma$ rotates around the axis $\ell$, it simultaneously displaces parallel lines orthogonal to the axis $\ell$, so that the speed of displacement is proportional to the speed of rotation. The resulting hypersurface is called the helicoidal hypersurface with axis $\ell$ and pitches $a, b \in \mathbb{R} \backslash\{0\}$.

We can suppose that $\ell$ is the line spanned by the vector $(0,0,0,1)^{t}$. The rotation matrix is given by

$$
Q(v, w)=\left(\begin{array}{cccc}
\cos v \cos w & -\sin v & -\cos v \sin w & 0 \\
\sin v \cos w & \cos v & -\sin v \sin w & 0 \\
\sin w & 0 & \cos w & 0 \\
0 & 0 & 0 & 1
\end{array}\right)
$$


where $v, w \in \mathbb{R}$. The matrix $Q$ supplies the following equations

$$
Q . \ell=\ell, Q^{t} \cdot Q=Q \cdot Q^{t}=\mathcal{I}_{4}, \operatorname{det} Q=1 .
$$

When the axis of rotation is $\ell$, there is an Euclidean transformation by which the axis is $\ell$ transformed to the $x_{4}$-axis of $\mathbb{E}^{4}$. Parametrization of the profile curve is given by $\gamma(u)=(u, 0,0, \varphi(u))$, where $\varphi(u): I \subset \mathbb{R} \longrightarrow \mathbb{R}$ is a differentiable function for all $u \in I$. Therefore, the helicoidal hypersurface, spanned by the vector $\ell=(0,0,0,1)$, is given as follows

$$
\mathcal{H}(u, v, w)=\underbrace{Q \cdot \gamma^{t}}_{\text {rotation }}+\underbrace{(a v+b w) \cdot \ell^{t}}_{\text {translation }}
$$

where $u \in I, v, w \in[0,2 \pi], a, b \in \mathbb{R} \backslash\{0\}$. We can also write the helicoidal hypersurface as follows

$$
\mathcal{H}(u, v, w)=\left(\begin{array}{c}
u \cos v \cos w \\
u \sin v \cos w \\
u \sin w \\
\varphi(u)+a v+b w
\end{array}\right) .
$$

When the pitches $a=b=0$, helicoidal hypersurface transforms into a rotational hypersurface in $\mathbb{E}^{4}$.

\section{Dini-Type Helicoidal Hypersurface and the Fourth Fundamental Form}

Next, for the sake of breviety, we use $\mathcal{S}_{u}=\sin u, \mathcal{C}_{u}=\cos u, \boldsymbol{T}_{u}=\tan u, \boldsymbol{C}_{u}=\cot u$. We consider Dini-type helicoidal hypersurface (see Figure 1) as follows

$$
\mathfrak{D}(u, v, w)=\left(\begin{array}{c}
\mathcal{S}_{u} \mathcal{C}_{v} \mathcal{C}_{w} \\
\mathcal{S}_{u} \mathcal{S}_{v} \mathcal{C}_{w} \\
\mathcal{S}_{u} \mathcal{S}_{w} \\
\mathcal{C}_{u}+\log \left(\boldsymbol{T}_{u / 2}\right)+a v+b w
\end{array}\right),
$$

where $u, a, b \in \mathbb{R} \backslash\{0\}$ and $0 \leq v, w \leq 2 \pi$.
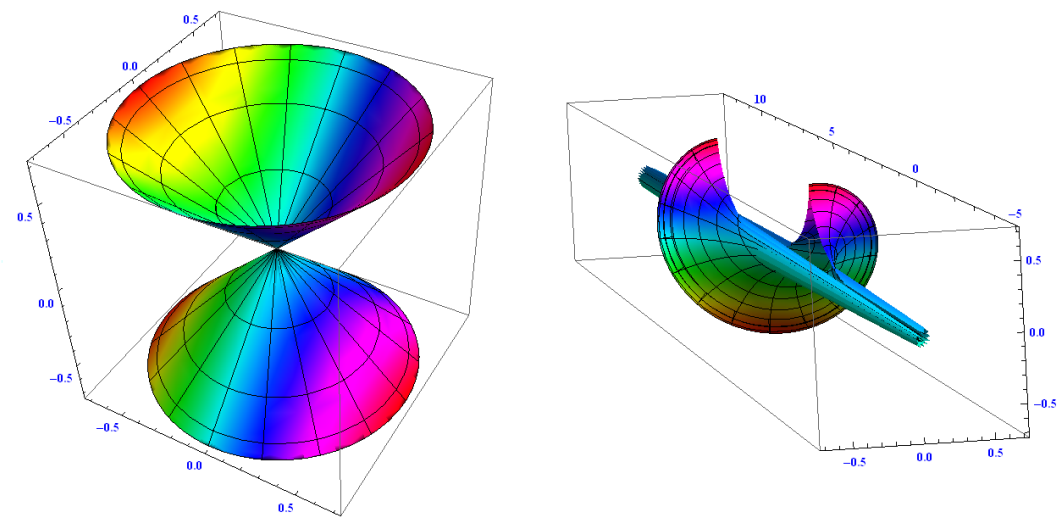

Figure 1. Projections of Dini-type hypersurface $\mathfrak{D}$ into (Left) $x_{1} x_{2} x_{3}$ space, (Right) $x_{1} x_{2} x_{4}$ space.

Using the first differentials of (1) with respect to $u, v, w$, we get the first quantities

$$
I=\left(\begin{array}{ccc}
C_{u}^{2} & a C_{u} \mathcal{C}_{u} & b \mathcal{C}_{u} \mathcal{C}_{u} \\
a C_{u} \mathcal{C}_{u} & \mathcal{S}_{u}^{2} \mathcal{C}_{w}^{2}+a^{2} & a b \\
b C_{u} \mathcal{C}_{u} & a b & \mathcal{S}_{u}^{2}+b^{2}
\end{array}\right)
$$

and its determinant

$$
\operatorname{det} I=\left(\left(b^{2}+1\right) \mathcal{C}_{w}^{2}+a^{2}\right) \mathcal{S}_{u}^{2} \mathcal{C}_{u}^{2}
$$


The Gauss map of (1) is given by

$$
\mathcal{G}=\frac{1}{W^{1 / 2}}\left(\begin{array}{c}
a \mathcal{S}_{v}-\left(b \mathcal{S}_{w}-\mathcal{C}_{u} \mathcal{C}_{w}\right) \mathcal{C}_{v} \mathcal{C}_{w} \\
a \mathcal{C}_{v}-\left(b \mathcal{S}_{w}-\mathcal{C}_{u} \mathcal{C}_{w}\right) \mathcal{S}_{v} \mathcal{C}_{w} \\
\left(b \mathcal{C}_{w}+\mathcal{C}_{u} \mathcal{S}_{w}\right) \mathcal{C}_{w} \\
-\mathcal{S}_{u} \mathcal{C}_{w}
\end{array}\right)
$$

where $W=\left(b^{2}+1\right) \mathcal{C}_{w}^{2}+a^{2}$. Taking the second differentials of (1) with respect to $u, v, w$, with (2), we have the second quantities as follows

$$
I I=\frac{1}{W^{1 / 2}}\left(\begin{array}{ccc}
\mathcal{C}_{u} \mathcal{C}_{w} & a \mathcal{C}_{u} \mathcal{C}_{w} & b \mathcal{C}_{u} \mathcal{C}_{w} \\
a \mathcal{C}_{u} \mathcal{C}_{w} & \left(b \mathcal{S}_{w}-\mathcal{C}_{u} \mathcal{C}_{w}\right) \mathcal{S}_{u} \mathcal{C}_{w}^{2} & -a \mathcal{S}_{u} \mathcal{S}_{w} \\
b \mathcal{C}_{u} \mathcal{C}_{w} & -a \mathcal{S}_{u} \mathcal{S}_{w} & -\mathcal{S}_{u} \mathcal{C}_{u} \mathcal{C}_{w}
\end{array}\right)
$$

Computing product matrix $I^{-1} . S$, we obtain the shape operator matrix of (1) as follows

$$
S=\frac{1}{W^{1 / 2}}\left(\begin{array}{ccc}
\frac{\mathcal{S}_{u} \mathcal{C}_{w}}{\mathcal{C}_{u}} & \frac{a \mathcal{C}_{w}}{\mathcal{C}_{u}} & \frac{a^{2}\left(b \mathcal{C}_{w}+\mathcal{C}_{u} \mathcal{S}_{w}\right)+b\left(b^{2}+1\right) \mathcal{C}_{w}^{3}}{W \mathcal{C}_{u}} \\
0 & \frac{b \mathcal{S}_{w}-\mathcal{C}_{u} \mathcal{C}_{w}}{\mathcal{S}_{u}} & -\frac{a\left(b^{2}+1\right) \mathcal{S}_{w}}{W \mathcal{S}_{u}} \\
0 & -\frac{a \mathcal{S}_{w}}{\mathcal{S}_{u}} & \frac{a^{2}\left(b \mathcal{S}_{w}-\mathcal{C}_{u} \mathcal{C}_{w}\right)-\left(b^{2}+1\right) \mathcal{C}_{u} \mathcal{C}_{w}^{3}}{W \mathcal{S}_{u}}
\end{array}\right) .
$$

Theorem 4. Let $\mathfrak{D}: M^{3} \longrightarrow \mathbb{E}^{4}$ be an immersion given by (1). Then, characteristic polynomial of $S$ is given as follows

$$
x^{3}+r x^{2}+s x+t=0
$$

where

$$
\begin{aligned}
& r=\frac{\left\{\begin{array}{c}
\left(b^{2}+1\right) \mathcal{C}_{u}^{2} \mathcal{C}_{w}^{3}-W \mathcal{S}_{u}^{2} \mathcal{C}_{w} \\
-\left(W+a^{2}\right)\left(b \mathcal{S}_{w}-\mathcal{C}_{u} \mathcal{C}_{w}\right) \mathcal{C}_{u}
\end{array}\right\}}{W^{3 / 2} \mathcal{S}_{u} \mathcal{C}_{u}}, \\
& s=\frac{\left\{\begin{array}{c}
+\left(W+a^{2}\right)\left(b \mathcal{S}_{w}-\mathcal{C}_{u} \mathcal{C}_{w}\right) \mathcal{S}_{u}^{2} \mathcal{C}_{w} \\
-2 a^{2} b \mathcal{C}_{u}^{2} \mathcal{S}_{w} \mathcal{C}_{w}+a^{2} \mathcal{C}_{u}\left(\mathcal{C}_{u}^{2} \mathcal{C}_{w}^{2}-\mathcal{S}_{w}^{2}\right) \\
+\left(b^{2}+1\right)\left(\mathcal{C}_{2 u} \mathcal{C}_{w}-b \mathcal{C}_{u} \mathcal{S}_{w}\right) \mathcal{C}_{u} \mathcal{C}_{w}^{3}
\end{array}\right\}}{W^{2} \mathcal{S}_{u}^{2} \mathcal{C}_{u}}, \\
& t=\frac{\left\{\begin{array}{c}
a^{2}\left(\mathcal{S}_{w}^{2}-\mathcal{C}_{u}^{2} \mathcal{C}_{w}^{2}\right)+2 a^{2} b \mathcal{C}_{u} \mathcal{S}_{w} \mathcal{C}_{w} \\
+\left(b^{2}+1\right)\left(b \mathcal{S}_{w}-\mathcal{C}_{u} \mathcal{C}_{w}\right) \mathcal{C}_{u} \mathcal{C}_{w}^{3}
\end{array}\right\} \mathcal{C}_{w}}{W^{5 / 2} \mathcal{S}_{u} \mathcal{C}_{u}}
\end{aligned}
$$

Proof. Computing $\operatorname{det}\left(S-x \mathcal{I}_{3}\right)=0$, we get $r, s$, and $t$.

Corollary 1. Let $\mathfrak{D}: M^{3} \longrightarrow \mathbb{E}^{4}$ be an immersion given by (1). Then, $\mathfrak{D}$ has the following principal curvatures

$$
\begin{aligned}
k_{1} & =\frac{\mathcal{S}_{u} \mathcal{C}_{w}}{W^{1 / 2} \mathcal{C}_{u}}, \\
k_{2} & =\frac{\wp^{1 / 2}-2 W \mathcal{C}_{u} \mathcal{C}_{w}+\left(W+a^{2}\right) b \mathcal{S}_{w}}{2 W^{3 / 2} \mathcal{S}_{u}}, \\
k_{3} & =\frac{-\wp^{1 / 2}-2 W \mathcal{C}_{u} \mathcal{C}_{w}+\left(W+a^{2}\right) b \mathcal{S}_{w}}{2 W^{3 / 2} \mathcal{S}_{u}},
\end{aligned}
$$


where

$$
\begin{aligned}
W= & \left(b^{2}+1\right) \mathcal{C}_{w}^{2}+a^{2} \\
\wp= & \left(4 a^{2} W+b^{2}\left(W+a^{2}\right)^{2}\right) \mathcal{S}_{w}^{2}+\left(b^{2}+1\right)^{2} \mathcal{C}_{u}^{2} \mathcal{C}_{w}^{6} \\
& +2\left(b^{2}+1\right)\left(W-a^{2}\right)\left(\mathcal{C}_{u} \mathcal{C}_{w}+b \mathcal{S}_{w}\right) \mathcal{C}_{u} \mathcal{C}_{w}^{3} \\
& +\left(a^{2}-W\right)^{2}\left(\mathcal{C}_{u} \mathcal{C}_{w}-2 b \mathcal{S}_{w}\right) \mathcal{C}_{u} \mathcal{C}_{w} .
\end{aligned}
$$

Proof. Solving characteristic polynomial of $S$, we obtain eigenvalues $k_{i}$.

Hence, we can see the curvatures of (1), using the following formulas

$$
\begin{aligned}
& \mathfrak{C}_{0}=1, \\
& \mathfrak{C}_{1}=\frac{k_{1}+k_{2}+k_{3}}{3}, \\
& \mathfrak{C}_{2}=\frac{k_{1} k_{2}+k_{1} k_{3}+k_{2} k_{3}}{3}, \\
& \mathfrak{C}_{3}=k_{1} k_{2} k_{3},
\end{aligned}
$$

easily. See [59] for the formulas of the curvatures $\mathfrak{C}_{i}$.

Corollary 2. Let $\mathfrak{D}: M^{3} \longrightarrow \mathbb{E}^{4}$ be an immersion given by (1). Then, (1) has the third fundamental form matrix as follows

$$
I I I=\left(\begin{array}{ccc}
\frac{\mathcal{C}_{w}^{2}}{W} & \frac{a \mathcal{S}_{u} \mathcal{C}_{w}^{2}}{W} & \frac{b \mathcal{S}_{u} \mathcal{C}_{w}^{2}}{W} \\
\frac{a \mathcal{S}_{u} \mathcal{C}_{w}^{2}}{W} & \frac{\left(b \mathcal{S}_{w}-\mathcal{C}_{u} \mathcal{C}_{w}\right)^{2} \mathcal{C}_{w}^{2}+a^{2}}{W} & \frac{a\left(b \mathcal{C}_{2 w}+\mathcal{C}_{u} \mathcal{S}_{2 w}\right)}{W} \\
\frac{b \mathcal{S}_{u} \mathcal{C}_{w}^{2}}{W} & \frac{a\left(b \mathcal{C}_{2 w}+\mathcal{C}_{u} \mathcal{S}_{2 w}\right)}{W} & x_{33}
\end{array}\right),
$$

where

$$
\begin{aligned}
W & =\left(b^{2}+1\right) \mathcal{C}_{w}^{2}+a^{2} \\
x_{33} & =\frac{a^{2}\left(b^{2}+1-\mathcal{C}_{w}^{2} \mathcal{S}_{w}^{2}\right)+\left(b^{2}+1\right)\left(b^{2}+\mathcal{C}_{u}^{2}\right) \mathcal{C}_{w}^{4}}{W}
\end{aligned}
$$

Proof. Using II.S of (1), we have the third fundamental form matrix.

Corollary 3. Let $\mathfrak{D}: M^{3} \longrightarrow \mathbb{E}^{4}$ be an immersion given by (1). Then, $\mathfrak{D}$ has the fourth fundamental form matrix

$$
I V=\frac{1}{W^{3 / 2}}\left(\begin{array}{lll}
f_{11} & f_{12} & f_{13} \\
f_{21} & f_{22} & f_{23} \\
f_{31} & f_{32} & f_{33}
\end{array}\right)
$$

where 


$$
\begin{aligned}
& W=\left(b^{2}+1\right) \mathcal{C}_{w}^{2}+a^{2}, \\
& f_{11}=\frac{\mathcal{S}_{u} \mathcal{C}_{w}^{3}}{\mathcal{C}_{u}}, \\
& f_{12}=f_{21}=\frac{a \mathcal{S}_{u}^{2} \mathcal{C}_{w}^{2}}{\mathcal{C}_{u}}, \\
& f_{13}=f_{31}=\frac{b \mathcal{S}_{u}^{2} \mathcal{C}_{w}^{2}}{\mathcal{C}_{u}}, \\
& f_{22}=\frac{\left\{\begin{array}{c}
a^{2}\left[\mathcal{S}_{u}^{2} \mathcal{C}_{w}^{2}-\left(1+2 \mathcal{S}_{w}^{2}\right) \mathcal{C}_{u}^{2}\right] \mathcal{C}_{w} \\
+2 a^{2} b \mathcal{C}_{u} \mathcal{S}_{w}^{3}+\left(b \mathcal{S}_{w}-\mathcal{C}_{u} \mathcal{C}_{w}\right)^{3} \mathcal{C}_{u} \mathcal{C}_{w}^{2}
\end{array}\right\}}{\mathcal{S}_{u} \mathcal{C}_{u}}
\end{aligned}
$$

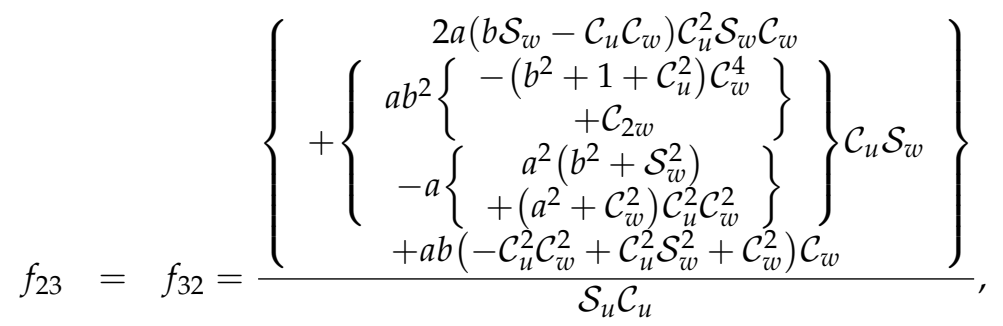

$$
\begin{aligned}
& \left\{\begin{array}{c}
-a^{2} b^{2}\left\{\begin{array}{c}
\left\{\begin{array}{c}
2\left(\mathcal{C}_{u}^{2}+1\right) \mathcal{C}_{u}^{2} \mathcal{C}_{w}^{4} \\
+2\left(\mathcal{C}_{w}^{2}+1\right) \mathcal{C}_{u}^{2} \mathcal{S}_{w}^{2}
\end{array}\right\} \mathcal{C}_{w} \\
+\left\{\begin{array}{c}
a^{2}\left(\mathcal{S}_{w}^{2}+1\right) \\
-b^{2}\left(2 \mathcal{C}_{w}^{2}+\mathcal{S}_{w}^{2}\right) \mathcal{C}_{w}^{2} \\
+\mathcal{S}_{u}^{2} \mathcal{C}_{w}^{2}
\end{array}\right\} \mathcal{C}_{u}^{2} \mathcal{C}_{w}
\end{array}\right\} \\
+a^{2} b\left\{\begin{array}{c}
b^{2}\left(2 b^{2}+\mathcal{C}_{u}^{2}+\mathcal{C}_{w}^{2}\right) \mathcal{C}_{w}^{4} \\
+a^{2}\left(1-\mathcal{S}_{u}^{2} \mathcal{C}_{w}^{2}+\left(b^{2}+1\right) \mathcal{S}_{w}^{2}\right. \\
+2 a^{2} b^{2}+\mathcal{S}_{u}^{2} \mathcal{C}_{u}^{2} \mathcal{C}_{w}^{2} \\
a^{2}\left(\mathcal{S}_{w}^{2}+\mathcal{C}_{u}^{2} \mathcal{C}_{w}^{2}\right) \\
+2 \mathcal{C}_{u}^{2} \mathcal{C}_{w}^{4}+\left(\mathcal{C}_{w}^{2}+2\right) \mathcal{S}_{w}^{2} \\
-\left(b^{2}+1\right)^{2}\left(b^{2}+\mathcal{C}_{u}^{2}\right) \mathcal{C}_{u}^{2} \mathcal{C}_{w}^{7} \\
+b^{2}\left(b^{2}+1\right) \mathcal{S}_{u}^{2} \mathcal{C}_{w}^{5}
\end{array}\right\} \mathcal{C}_{u}^{2} \mathcal{C}_{w} \\
W \mathcal{S}_{u} \mathcal{C}_{u}
\end{array}\right.
\end{aligned}
$$

Proof. Using product matrix III.S of (1), we get the fourth fundamental form matrix.

Example 1. When $a=b=0$ in hypersurface (1), we obtain Dini-type rotational hypersurface (see Figure 2) as follows

$$
\Re(u, v, w)=\left(\begin{array}{c}
\mathcal{S}_{u} \mathcal{C}_{v} \mathcal{C}_{w} \\
\mathcal{S}_{u} \mathcal{S}_{v} \mathcal{C}_{w} \\
\mathcal{S}_{u} \mathcal{S}_{w} \\
\mathcal{C}_{u}+\log \left(T_{u / 2}\right)
\end{array}\right) .
$$

Then its fundamental form matrices I, II, Gauss map $\mathcal{G}$ (see Figure 3), shape operator matrix $S$, fundamental form matrices $I I I, I V$, and curvatures $\mathfrak{C}_{i}$ are given by as follows 


$$
\begin{aligned}
I & =\operatorname{diag}\left(\boldsymbol{C}_{u}^{2}, \mathcal{S}_{u}^{2} \mathcal{C}_{w}^{2}, \mathcal{S}_{u}^{2}\right) \\
I I & =\operatorname{diag}\left(\boldsymbol{C}_{u},-1 / 2 \mathcal{S}_{2 u} \mathcal{C}_{w}^{2},-1 / 2 \mathcal{S}_{2 u}\right), \\
\mathcal{G} & =\left(\mathcal{C}_{u} \mathcal{C}_{v} \mathcal{C}_{w}, \mathcal{C}_{u} \mathcal{S}_{v} \mathcal{C}_{w}, \mathcal{C}_{u} \mathcal{S}_{w},-\mathcal{S}_{u}\right), \\
S & =\operatorname{diag}\left(\boldsymbol{T}_{u},-\mathcal{C}_{u},-\boldsymbol{C}_{u},\right) \\
I I I & =\operatorname{diag}\left(1, \mathcal{C}_{u}^{2} \mathcal{C}_{w}^{2}, \mathcal{C}_{u}^{2}\right), \\
I V & =\operatorname{diag}\left(\boldsymbol{T}_{u},-\mathcal{C}_{u} \mathcal{C}_{u}^{2} \mathcal{C}_{w,}^{2},-\boldsymbol{C}_{u} \mathcal{C}_{u}^{2}\right),
\end{aligned}
$$

and

$$
\begin{aligned}
& \mathfrak{C}_{0}=1, \\
& \mathfrak{C}_{1}=1 / 3 T_{u}-2 / 3 C_{u}, \\
& \mathfrak{C}_{2}=C_{u}^{2}-2 / 3 \mathcal{S}_{u}^{-2}, \\
& \mathfrak{C}_{3}=C_{u},
\end{aligned}
$$

where $\mathcal{S}_{u}=\sin u, \mathcal{C}_{u}=\cos u, T_{u}=\tan u, C_{u}=\cot u$.
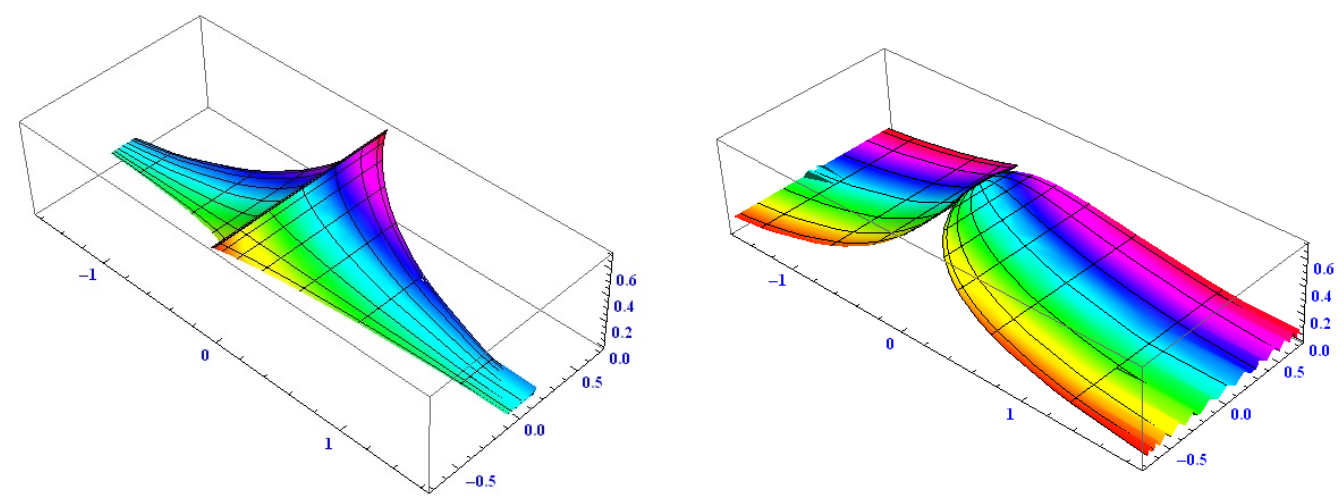

Figure 2. Projections of $\mathfrak{R}(u, v, w)$ into (Left) $x_{1} x_{3} x_{4}$ space, (Right) $x_{2} x_{3} x_{4}$ space.
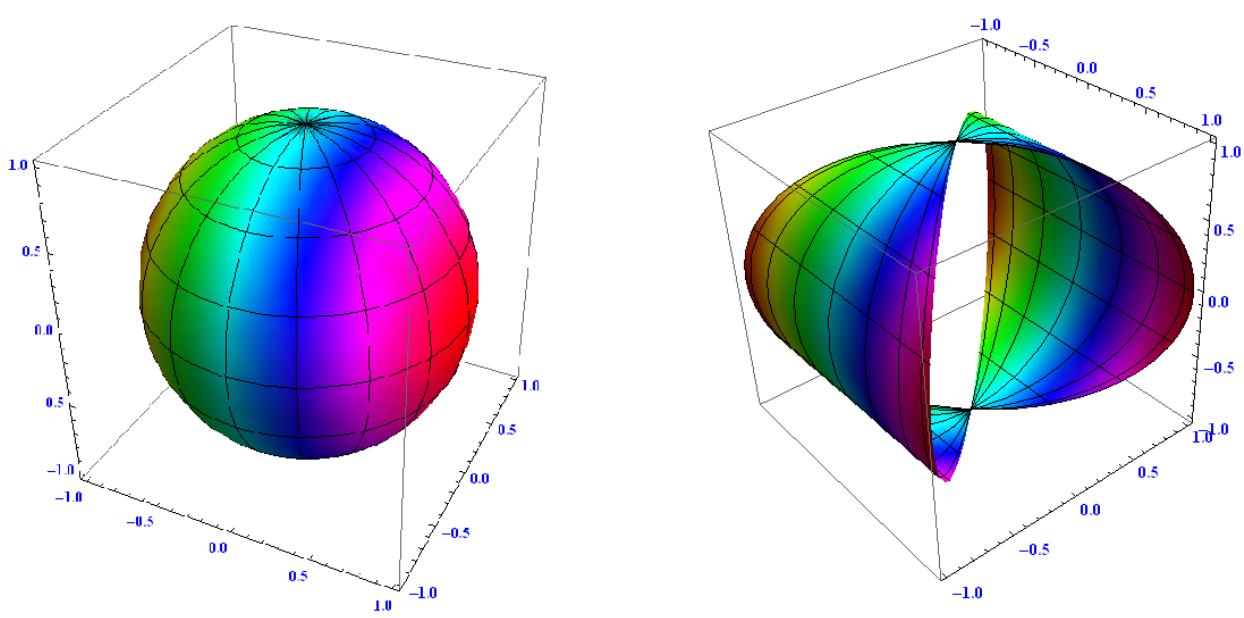

Figure 3. Projections of $\mathcal{G}$ of $\Re$ into (Left) $x_{1} x_{3} x_{4}$ space, (Right) $x_{2} x_{3} x_{4}$ space.

\section{Conclusions}

In this paper, we introduce the fourth fundamental form of Dini-type helicoidal hypersurface $\mathfrak{D}(u, v, w)$ in the four dimensional Euclidean space $\mathbb{E}^{4}$. We compute its Gauss 
map $\mathcal{G}$. We obtain the characteristic polynomial of shape operator matrix $S$. We calculate the fourth fundamental form matrix $I V$ of hypersurface $\mathfrak{D}$. Taking pitches $a=b=0$ of helicoidal hypersurface $\mathfrak{D}$, we have a Dini-type rotational hypersurface $\mathfrak{R}(u, v, w)$, and reveal its differential geometric objects. Therefore, it can be seen that objects of $\mathfrak{D}$ and $\mathfrak{R}$ supply the following relation $\mathfrak{C}_{0} I V-3 \mathfrak{C}_{1} I I I+3 \mathfrak{C}_{2} I I-\mathfrak{C}_{3} I=0$.

Funding: This work received no external funding.

Acknowledgments: The author would like to express their sincere thanks to the editor and the reviewers for their helpful comments and suggestions.

Conflicts of Interest: The author declares that no conflict of interest of the paper.

\section{References}

1. Euler, L. Methodus Inveniendi Lineas Curvas Maximi Minimive Propietate Gaudeates Sive Solutio Problematis Isoperimetrici Latissimo Sensu Accepti; Opera Omnia Citation: Series 1 Original Source Citation; Apud Marcum-Michaelem Bousquet \& Socios: Genevæ, Switzerland, 1744; Volume 24, pp. 1-322.

2. Meusnier, J.B. Memoire sur la courbure des surfaces. Mem. Mathem. Phys. Acad. Sci. Paris, Pres. par Div. Savans 1785, 10, 477-510; Presented in 1776.

3. Bour, E. Théorie de la déformation des surfaces. J. l.Êcole Imp. Polytech. 1862, 22, 1-148.

4. Dini, U. Sopra le funzioni di una variabile complessa. Ann. Di Matematica Pura Appl. 1871, 4, 159-174. [CrossRef]

5. Moore, C. Surfaces of rotation in a space of four dimensions. Ann. Math. 1919, 21, 81-93. [CrossRef]

6. Moore, C. Rotation surfaces of constant curvature in space of four dimensions. Bull. Amer. Math. Soc. 1920, 26, 454-460. [CrossRef]

7. Do Carmo, M.; Dajczer, M. Helicoidal surfaces with constant mean curvature. Tohoku Math. J. 1982, 34, 351-367. [CrossRef]

8. Chen, B.Y. Total Mean Curvature and Submanifolds of Finite Type; World Scientific: Singapore, 1984.

9. Hano, J.; Nomizu, K. Surfaces of revolution with constant mean curvature. Tohoku Math. J. 1984, 36, 427-437. [CrossRef]

10. Roussos, I.M. The helicoidal surfaces as Bonnet surfaces. Tohoku Math. J. 1988, 40, 485-490. [CrossRef]

11. Ripoll, J.B. Helicoidal minimal surfaces in hyperbolic space. Nagoya Math. J. 1989, 114, 65-75. [CrossRef]

12. Dillen, F. Ruled submanifolds of finite type. Proc. Am. Math. Soc. 1992, 114, 795-798. [CrossRef]

13. Baikoussis, C.; Verstraelen, L. On the Gauss map of helicoidal surfaces. Rend. Sem. Mat. Messina Ser. II 1993, 2, 31-42.

14. Hoffman, D.; Wei, F.S.; Karcher, H. Adding handles to the helicoid. Bull. Amer. Math. Soc. 1993, 29, 77-84. [CrossRef]

15. Gray, A. Modern Differential Geometry of Curves and Surfaces with Mathematica, 2nd ed.; CRC Press: Boca Raton, FL, USA, 1997; pp. 493-495.

16. Baikoussis, C.; Koufogiorgos, T. Helicoidal surfaces with prescribed mean or Gaussian curvature. J. Geom. 1998, 63, 25-29. [CrossRef]

17. Dillen, F.; Kühnel, W. Ruled Weingarten surfaces in Minkowski 3-space. Manuscripta Math. 1999, 98, 307-320. [CrossRef]

18. Ikawa, T. Bour's theorem and Gauss map. Yokohama Math. J. 2000, 48, 173-180.

19. Sasahara, N. Spacelike helicoidal surfaces with constant mean curvature in Minkowski 3-space. Tokyo J. Math. 2000, 23, 477-502. [CrossRef]

20. Ikawa, T. Bour's theorem in Minkowski geometry. Tokyo J. Math. 2001, 24, 377-394. [CrossRef]

21. Choi, M.; Kim, Y.H. Characterization of the helicoid as ruled surfaces with pointwise 1-type Gauss map. Bull. Korean Math. Soc. 2001, 38, 753-761.

22. Yoon, D.W. Rotation surfaces with finite type Gauss map in $\mathbb{E}^{4}$. Indian J. Pure Appl. Math. 2001, 32, $1803-1808$.

23. Beneki, C.C.; Kaimakamis, G.; Papantoniou, B.J. Helicoidal surfaces in three-dimensional Minkowski space. J. Math. Anal. Appl. 2002, 275, 586-614. [CrossRef]

24. Güler, E.; Vanl1, A. Bour's theorem in Minkowski 3-space. J. Math. Kyoto 2006, 46, 47-63. [CrossRef]

25. Güler, E.; Vanlı, A. On the mean, Gauss, the second Gaussian and the second mean curvature of the helicoidal surfaces with light-like axis in $\mathbb{R}_{1}^{3}$. Tsukuba J. Math. 2008, 32, 49-65. [CrossRef]

26. Stamatakis, S.; Al-Zoubi, H. Surfaces of revolution satisfying $\Delta^{I I I} x=$ Ax. J. Geom. Graph. 2010, 14, 181-186.

27. Ji, F.; Kim, Y.H. Helicoidal CDPC-surfaces in Minkowski 3-space. Int. J. Geom. Methods Mod. Phys. 2010, 7, 979-988. [CrossRef]

28. Ji, F.; Kim, Y.H. Mean curvatures and Gauss maps of a pair of isometric helicoidal and rotation surfaces in Minkowski 3-space. J. Math. Anal. Appl. 2010, 368, 623-635. [CrossRef]

29. Güler, E.; Yaylı, Y.; Hacisalihoğlu, H.H. Bour's theorem on the Gauss map in 3-Euclidean space. Hacet. J. Math. Stat. 2010, 39, 515-525.

30. Arslan, K.; Kılıç Bayram, B.; Bulca, B.; Kim, Y.H.; Murathan, C.; Öztürk, G. Rotational embeddings in $\mathbb{E}^{4}$ with pointwise 1-type Gauss map. Turk. J. Math. 2011, 35, 493-499.

31. Dursun, U.; Turgay, N.C. General rotational surfaces in Euclidean space $\mathbb{E}^{4}$ with pointwise 1-type Gauss map. Math. Commun. 2012, 17, 71-81.

32. Arslan, K.; Bayram, B.; Bulca, B.; Öztürk, G. Generalized rotation surfaces in $\mathbb{E}^{4}$. Results Math. 2012, 61, 315-327. [CrossRef] 
33. Dursun, U.; Turgay, N.C. Minimal and pseudo-umbilical rotational surfaces in Euclidean space $\mathbb{E}^{4}$. Mediterr. J. Math. 2013, 10, 497-506. [CrossRef]

34. Perdomo, O.M. Helicoidal minimal surfaces in $\mathbb{R}^{3}$. Illinois J. Math. 2013, 57, 87-104. [CrossRef]

35. Ji, F.; Kim, Y.H. Isometries between minimal helicoidal surfaces and rotation surfaces in Minkowski space. Appl. Math. Comput. 2013, 220, 1-11. [CrossRef]

36. Kim, Y.H.; Turgay, N.C. Surfaces in $\mathbb{E}^{4}$ with $L_{1}$-pointwise 1-type Gauss map. Bull. Korean Math. Soc. 2013, 50, 935-949. [CrossRef]

37. Kim, Y.H.; Turgay, N.C. Classifications of helicoidal surfaces with $L_{1}$-pointwise 1-type Gauss map. Bull. Korean Math. Soc. 2013, 50, 1345-1356. [CrossRef]

38. Güler, E. A new kind of helicoidal surface of value m. Int. Electron. J. Geom. 2014, 7, 154-162. [CrossRef]

39. López, R.; Demir, E. Helicoidal surfaces in Minkowski space with constant mean curvature and constant Gauss curvature. Cent. Eur. J. Math. 2014, 12, 1349-1361. [CrossRef]

40. Arslan, K.; Bulca, B.; Milousheva, V. Meridian surfaces in $\mathbb{E}^{4}$ with pointwise 1-type Gauss map. Bull. Korean Math. Soc. 2014, 51, 911-922. [CrossRef]

41. Ganchev, G.; Milousheva, V. General rotational surfaces in the 4-dimensional Minkowski space. Turk. J. Math. 2014, 38, 883-895. [CrossRef]

42. Babaarslan, M.; Yayl1, Y. Space-like loxodromes on rotational surfaces in Minkowski 3-space. J. Math. Anal. Appl. 2014, 409, 288-298. [CrossRef]

43. Güler, E.; Yaylı, Y. Generalized Bour's theorem. Kuwait J. Sci. 2015, 42, 79-90.

44. Senoussi, B.; Bekkar, M. Helicoidal surfaces with $\Delta^{J} r=A r$ in 3-dimensional Euclidean space. Stud. Univ. Babeş-Bolyai Math. 2015, $60,437-448$.

45. Hoffman, D.; Traizet, M.; White, B. Helicoidal minimal surfaces of prescribed genus. Acta Math. 2016, 216, 217-323. [CrossRef]

46. Kim, D.S.; Kim, J.R.; Kim, Y.H. Cheng-Yau operator and Gauss map of surfaces of revolution. Bull. Malays. Math. Sci. Soc. 2016, 39, 1319-1327. [CrossRef]

47. Güler, E.; Magid, M.; Yayl, Y. Laplace Beltrami operator of a helicoidal hypersurface in four space. J. Geom. Symmetry Phys. 2016, 41,77-95. [CrossRef]

48. Hieu, D.T.; Thang, N.N. Bour's theorem in 4-dimensional Euclidean space. Bull. Korean Math. Soc. 2017, 54, $2081-2089$.

49. Arslan, K.; Bulca, B.; Kosova, D. On generalized rotational surfaces in Euclidean spaces. J. Korean Math. Soc. 2017, 54, 999-1013. [CrossRef]

50. Babaarslan, M.; Kayacık, M. Time-like loxodromes on helicoidal surfaces in Minkowski 3-space. Filomat 2017, 31, 4405-4414. [CrossRef]

51. Güler, E.; Hacısalihoğlu, H.H.; Kim, Y.H. The Gauss map and the third Laplace-Beltrami operator of the rotational hypersurface in 4-space. Symmetry 2018, 10, 398. [CrossRef]

52. Aleksieva, Y.; Milousheva, V.; Turgay, N.C. General rotational surfaces in pseudo-Euclidean 4-space with neutral metric. Bull. Malays. Math. Sci. Soc. 2018, 41, 1773-1793. [CrossRef]

53. Goemans, W. Flat double rotational surfaces in Euclidean and Lorentz-Minkowski 4-space. Publ. Inst. Math. 2018, 103, 61-68. [CrossRef]

54. Güler, E.; Turgay, N.C. Cheng-Yau operator and Gauss map of rotational hypersurfaces in 4-space. Mediterr. J. Math. 2019, 16, 1-16. [CrossRef]

55. Güler, E.; Kişi, Ö. Dini-type helicoidal hypersurfaces with timelike axis in Minkowski 4-space $\mathbb{E}_{1}^{4}$. Mathematics 2019, 7, 205. [CrossRef]

56. Yoon, D.W.; Lee, J.W.; Lee, C.W. Constructions of helicoidal surfaces by using curvature functions in isotropic space. Hacet. J. Math. Stat. 2019, 48, 959-965. [CrossRef]

57. Güler, E. Helical hypersurfaces in Minkowski geometry $\mathbb{E}_{1}^{4}$. Symmetry 2020, 12, 1206. [CrossRef]

58. Dursun, U. Rotational Weingarten surfaces in hyperbolic 3-space. J. Geom. 2020, 111, 1-12. [CrossRef]

59. Güler, E. Fundamental form $I V$ and curvature formulas of the hypersphere. Malaya J. Mat. 2020, 8, 2008-2011. [CrossRef]

60. López, R.; Pámpano, Á. Classification of rotational surfaces with constant skew curvature in 3-space forms. J. Math. Anal. Appl. 2020, 489, 1-19. [CrossRef] 